healthcare workers to recommendations for hand hygiene remains low. To improve the rate of adherence with hand hygiene, researchers from Cook County Hospital installed sinkless, alcohol-based degermers and delivered inservice education to hospital personnel using an interactive audience response (AR) system.

The researchers conducted 479 hours of direct hand hygiene observations on 24 patient-care units at 4 facilities during a 14-month period. Adherence to hand hygiene was defined as any act of hand antisepsis with soap and water or the alcohol-based degermer after patient contact. A 40minute AR presentation was developed that contained questions related to hand hygiene and included educational slides on effectiveness, convenience, and benefits to skin of sinkless degermers.

Among the 4 study hospitals, overall adherence to hand hygiene was 37\%. Hospital-specific rates varied considerable among the 4 facilities (range, 26\% to $46 \%$ ). Rates of hand hygiene after patient contact increased significantly in hospitals A and B. In hospital C, the rate of hand hygiene remained stable, but there was an increase in the use of the alcohol-based degermer. At hospital D, which did not receive the $\mathrm{AR}$ intervention, the adherence with hand hygiene remained low and showed no significant change over time.

The researchers concluded that the introduction of sinkless degermers in conjunction with an interactive educational inservice resulted in increased adherence with hand hygiene.

FROM: Vernon MO, Peterson BJ, Welbel SF, Trick WE, Weinstein RA. Impact of an interactive educational intervention on hand hygiene adherence rates in a multicenter study. Presented at the 41st Annual Interscience Conference on Antimicrobial Agents and Chemotherapy; December 16-19, 2001; Chicago, IL. Abstract no. K1331.

\section{Rings as a Risk Factor for Hand Colonization in an Intensive Care Unit}

Risk factors for potential pathogens on the hands of registered nurses were studied in a 27-bed surgical intensive care unit. Using a glove juice technique, Hayes and colleagues from Cook County Hospital, the Centers for Disease Control and Prevention, and Rush Medical College sampled the hands of 66 nurses during 14 weeks. Potential risk factors were skin condition, dominant hand, glove use, ring wear, nail length, nail application, and number of assigned patients.

The nurses' hands were found to be contaminated with methicillin-resistant coagulase-negative staphylococci (71\%), gram-negative bacilli (15\%), Staphylococcus aureus (14\%), Candida species (12\%), and vancomycin-resistant enterococci (2.1\%). With the use of univariate analysis, several risk factors were identified for hand contamination. With the use of multivariate analysis, only ring wear remained an independent risk factor for contamination by presumably transient organisms (ie, methicillin-resistant coagulase-negative staphylococci excluded) regardless of the category of organism. The colonization frequencies of those who did and those who did not wear rings were $25 \%$ and $9.5 \%$ for gram-negative bacilli, $25 \%$ and $9.5 \%$ for $S$. aureus, and $21 \%$ and $8.5 \%$ for Candida species. With ring wear, there was a stepwise increase in hand contamination by any transient organism: no rings, 29\%; 1 ring, 76\%; and more than 1 ring, 94\%. Ring wear was also associated with a greater median number of colony-forming units for gramnegative bacilli ( 325 for ring wear vs 50 for no rings) and Candida species (120 for ring wear vs 10 for no rings).

The researchers concluded that wearing rings was a major risk factor for hand carriage of many potential pathogens.

FROM: Hayes RA, Trick WE, Vernon MO, et al. Ring use as a risk factor for hand colonization in a surgical intensive care unit. Presented at the 41st Annual Interscience Conference on Antimicrobial Agents and Chemotherapy; December 16-19, 2001; Chicago, IL. Abstract no. K-1333.

\section{Vancomycin Versus Cefazolin Prophylaxis for Cardiac Surgery When the Prevalence of Methicillin-Resistant Staphylococcal Infections Is High}

Finkelstein and colleagues from Haifa, Israel, conducted a study to compare the efficacy of vancomycin prophylaxis with that of cefazolin prophylaxis in preventing surgical-site infections in a tertiary-care medical center with a high prevalence of methicillin-resistant staphylococcal infections. All patients 18 years and older scheduled for cardiac surgery requiring sternotomy were randomly assigned to receive vancomycin (1 g every 12 hours) or cefazolin ( $1 \mathrm{~g}$ every 8 hours). Prophylaxis was started during the induction of anesthesia and continued for only 24 hours. Patients were followed for at least 30 days ( 1 year for those receiving a cardiac implant). Surgical-site infections were stratified according to the National Nosocomial Infections Surveillance System risk index.

Of the 885 patients included in the study, 452 received vancomycin and 433 received cefazolin. The overall surgical-site infection rates were similar in the two groups (43 cases in the vancomycin group [9.5\%] vs 39 cases in the cefazolin group [9.0\%], $P=.8$ ). Superficial and deep incisional surgical-site infection rates were also similar in the two groups. There was a trend toward more frequent organ-space infections and infections with beta-lactamresistant organisms among patients receiving cefazolin, but this trend did not reach statistical significance. In contrast, surgical-site infections caused by methicillin-susceptible staphylococci were significantly more common in the group receiving vancomycin (17 cases [3.7\%] vs 6 cases [1.3\%], $P=.04$ ). The durations of postoperative hospitalization and the mortality rates were similar in the two groups.

The authors concluded that this trial suggests that vancomycin and cefazolin have similar efficacies in preventing surgical-site infections in cardiac surgery.

FROM: Finkelstein R, Rabino G, Mashiah $T$, et al. 